\title{
Herramientas tecnológicas y su uso en la Universidad del Valle sede Buga
}

\section{Technological tools and their use in the Universidad del Valle sede Buga}

\author{
Luis Lasso-Cardona \\ Universidad del Valle sede Buga, Colombia \\ Giorman Rodríguez-Muñoz (D) \\ Universidad del Valle sede Buga, Colombia \\ Julián Andrés Llanos-Betancourt (D) \\ Universidad del Valle sede Buga, Colombia
}

\section{Resumen}

Objetivo: Evaluar el grado de uso de las herramientas tecnológicas ofrecidas por la Universidad del Valle en la sede de Buga, en los procesos de enseñanza y aprendizaje. Métodos: La investigación es de tipo cuantitativo y descriptivo. Se determinó el tamaño de muestra utilizando el muestreo de cuotas y se aplicó una encuesta a 313 estudiantes. Resultados: a) El 96\% consideran que el uso de las TIC es fundamental en su formación profesional, b) el $61 \%$ considera que los docentes capacitan y motivan a los estudiantes para el uso de herramientas tecnológicas en los procesos de aprendizaje, c) respecto a la frecuencia de uso, el correo institucional con un $60 \%$ es el más utilizado. La caja de herramientas y las bases de datos bibliográficas son las de más baja utilización, d) se identificó que el software licenciado tiene un porcentaje de uso muy bajo, y d) para el $96 \%$ de los estudiantes las TIC tienen mucha importancia en los procesos educativos. Conclusiones y discusiones: La Universidad del Valle ofrece gran cantidad de herramientas tecnológicas, pero que son inutilizadas y desconocidas. Se deben establecer estrategias en cuanto al uso de estas y brindar capacitación a los estudiantes y docentes para involucrarlos.

Palabras clave: TIC, educación, herramienta tecnológica, conocimiento.

\section{Abstract}

Objective: Evaluate the degree of use of the technological tools offered by the Universidad del Valle at the Buga headquarters, in the teaching and learning processes. Methods: The research is quantitative and descriptive. The sample size was determined using quota sampling and a survey was applied to 313 students. Results: a) $96 \%$ believe that the use of ICTs is essential in their professional training, b) $61 \%$ believe that teachers train and motivate students to use technological tools in learning processes, c) Regarding the frequency of use, institutional mail with $60 \%$ is the most used. The Toolbox and the Bibliographic Databases are the ones with the lowest use, d) it was identified that the licensed software has a very low percentage of use, and d) for $96 \%$ of the students, ICTs are very important in the processes Educational. Conclusions and discussions: Universidad del Valle offers a large number of technological tools, but they are unused and unknown. Strategies should be established regarding the use of these and provide training to students and teachers to involve them.
Open Access:

ISSN: $0124-2121$ E-ISSN: $2665-2420$

ARTÍCULO DE INVESTIGACIÓN CIENTÍFICA Copyright $\odot$ By Educación y Humanismo

Editor: Dhayana Fernández Matos Universidad Simón Bolívar

Correspondencia: Luis Lasso luis.lasso@correounivalle.edu.co

Keywords: ICT, education, technological tool, knowledge 


\section{Introducción}

Actualmente la sociedad está viviendo una serie de cambios por cuenta de la implementación de nuevas herramientas tecnológicas que afectan, de una u otra forma, la manera como se realizan las actividades diarias. Estos cambios están soportados en el uso de Tecnologías de la Información y Comunicación (TIC) (Islas, 2017), originando un proceso de transformación e innovación tecnológica que ha iniciado la generación de nuevo conocimiento, acelerándola globalización en contextos como el productivo, económico y social (Quiroga, Torrent y Murcia, 2017).

En este sentido, en los últimos años las TIC se han transformado en herramientas fundamentales en la educación al punto en que en la actualidad no se puede hablar de educación sin incluir a la tecnología, ya que esta brinda la capacidad de desarrollar conocimiento de una forma mucho más veloz y eficaz que en el modelo tradicional de enseñanza impartida por el docente (Abarca, 2015), promoviendo el desarrollo de competencias y habilidades prácticas por parte de los estudiantes, como es el caso en el uso de laboratorios virtuales que simulan situaciones reales en las empresas (Castro, Guzmán y Casado, 2007).

En el contexto educativo, el auge de las TIC está cambiando las metodologías convencionales de los procesos de enseñanza y de aprendizaje, afectando de manera directa a docentes y estudiantes y, por consiguiente, a la sociedad. Se exigen metodologías que estén en concordancia con nuevas tecnologías que implanten mejores formas de comunicar, divulgar, consultar y procesar el gran volumen de datos a disposición (Lasso y Sánchez-Medina, 2019). Por tal motivo, resulta válido argumentar que debe existir una estrecha correlación entre diversos aspectos como la enseñanza, conocimiento, innovación y nuevas TIC, entendiendo como estas han ayudado a la transformación de este escenario (Garcés, Garcés y Alcívar, 2016), y de esta forma realizar un análisis al interior de las instituciones educativas, de los docentes y los estudiantes que permita replantear las políticas y currículos educativos, y los métodos de enseñanza y aprendizaje.

En la educación, las TIC han forjado un nuevo ambiente en el cual el estudiante se ha convertido en un nuevo agente educativo empoderado de su proceso de aprendizaje, donde factores como el tiempo, la flexibilidad y la disponibilidad de la información, plantean nuevos paradigmas educativos y pedagógicos (Hernández, 2017). En este panorama, La Organización de las Naciones Unidas para la Educación, la Ciencia y la Cultura (UNESCO) desarrolló el programa de Estándares de Competencias en TIC para docentes (2008), dirigido a perfeccionar la práctica docente de manera global, teniendo en cuenta las capacidades en TIC para innovar los procesos de pedagogía, currículo y la gestión de las instituciones (Bustos y Parra, 2019). Es por ello por lo que, la integración de nuevas TIC en el ámbito educativo se muestra como una necesidad, en la cual las instituciones juegan un papel fundamental creando nuevas políticas que fomenten la implantación y uso de herramientas tecnológicas en pro de mejorar los procesos pedagógicos (Severin, 2010). 
Son estas razones las que explican el interés de la investigación que se presenta a continuación, la cual tiene como objetivo evaluar el grado de uso que los docentes y estudiantes le están dando a las herramientas tecnológicas educativas ofrecidas por la Universidad del Valle, en especial en la sede de Buga, en los procesos de enseñanza y aprendizaje en los cursos presenciales ofrecidos por la institución.

\section{Metodología}

\section{Tipo de investigación}

La investigación es de tipo cuantitativo y descriptivo, se seleccionó una serie de cuestiones y se midieron para describir lo que se investiga (Hernández, Fernández, \& Baptista, 2014). El trabajo se dividió en cuatro fases: (1) Se realizó una revisión de literatura recurriendo a las bases de datos Web of Science (WoS), SCOPUS, Latindex, para encontrar revistas científicas que permitieran entender el concepto de TIC y su relación con la educación; (2) Se analizaron las herramientas tecnológicas ofrecidas por la Universidad del Valle, para conocer sus características y utilidad; (3) Se diseñó y aplicó una encuesta dirigida a los estudiantes de las cinco facultades presentes en la sede Buga, para conocer la percepción que tienen los estudiantes acerca del uso de las TIC y de las herramientas ofrecidas por la Universidad del Valle. Las variables que se tomaron en consideración en la encuesta fueron: valoración en la formación profesional, incentivo en el uso de las herramientas tecnológicas proporcionadas, nivel de uso de recursos TIC en sus prácticas estudiantiles, valoración de la formación recibida para el uso de las TIC, percepción que existe en torno a las TIC y evaluación alrededor del empleo de las TIC en los procesos de enseñanza y aprendizaje; y (4) Se analizaron los resultados de la encuesta, los cuales fundamentaron las conclusiones.

\section{Población}

La Universidad del Valle es una institución pública de educación superior de Colombia, localizada en el Valle del Cauca y considerada como una de las más importantes del país. Su campus principal se encuentra en la ciudad de Cali y cuenta con ocho sedes regionales en el departamento y una en el Norte del Cauca.

La investigación se llevó a cabo en la sede Buga, en la cual funcionan cinco facultades discriminadas así: Facultad de Ingeniería compuesta por los programas de Ingeniería Industrial, Tecnología en Sistemas de Información y Tecnología en Electrónica; Facultad de Ciencias de la Administración conformada por los programas de Administración de Empresas, Contaduría Pública y Tecnología en Dirección de Empresas Turísticas; Facultad de Artes Integradas conformada por Comunicación Social y Periodismo y Licenciatura en Música; Facultad de Humanidades conformada por Licenciatura en Literatura y Licenciatura en Historia y, por último, el Instituto de Psicología. 
A la fecha de la investigación, la sede contaba con 1657 estudiantes inscritos en las cinco facultades de los diferentes programas de formación tecnológica y profesional.

Para determinar el tamaño de muestra adecuado para la recolección de datos se utilizó la fórmula (1):

$$
M=\frac{\frac{z^{2} \times p(1-p)}{e^{2}}}{1+\left(\frac{z^{2} \times(1-p)}{e^{2} N}\right)}
$$

Donde:

$N($ tamaño de población $)=1657$

$e$ (margen de error) $=5 \%$

$p$ (nivel de confianza deseado $)=95 \%$

$z$ (cantidad de desviaciones estándar) que según $p$ debe ser $=1.96$

Al aplicar la fórmula (1), da como resultado que el tamaño de la muestra $M=313$, que representa la cantidad de encuestas a aplicar.

\section{Tipo y tamaño de muestreo}

Para la recolección de datos se utilizó el muestreo de cuotas, el cual es frecuentemente usado en todo tipo de encuestas que buscan examinar la opinión de una población objetivo acerca de un tema en particular, fijando tamaños de los diferentes segmentos que la componen, estableciendo la cuota de entrevistas que se debe cubrir por cada grupo de la población (Pimienta, 2000).

Una vez se obtuvo el tamaño de la muestra $M$, se procedió a calcular el tamaño de cuota de cada grupo de la población teniendo en cuenta la cantidad de estudiantes por facultad. La tabla 1 resume el proceso realizado.

Tabla 1.

Tamaño de cuota por facultad.

\begin{tabular}{lccc}
\hline \multicolumn{1}{c}{ Facultades } & $\begin{array}{c}\text { \# Estudiantes x } \\
\text { Facultad }\end{array}$ & $\begin{array}{c}\text { Representación } \\
\text { Porcentaje }\end{array}$ & $\begin{array}{c}\text { \# Estudiantes a } \\
\text { encuestar } \\
\text { (Tamaño cuota) }\end{array}$ \\
\hline $\begin{array}{l}\text { Ingeniería (Ing. Industrial, Tec. } \\
\text { Sistemas, Tec. Electrónica) }\end{array}$ & 407 & $25 \%$ & 77 \\
\hline $\begin{array}{l}\text { Ciencias de la Administración } \\
\text { (Admón. Empresas, Contaduría }\end{array}$ & 668 & $40 \%$ & 126 \\
$\begin{array}{l}\text { Pública, Tec. Dirección de } \\
\text { Empresas Turísticas) }\end{array}$ & 156 & $9 \%$ & 29 \\
\hline $\begin{array}{l}\text { Instituto de Psicología (Psicología) } \\
\text { Artes Integradas (Comunicación }\end{array}$ & 426 & $26 \%$ & 80 \\
$\begin{array}{l}\text { Social y Periodismo, Música) y } \\
\text { Humanidades (Lic. en Literatura, } \\
\text { Lic. en Historia) }\end{array}$ & & & \\
\hline
\end{tabular}




\begin{tabular}{llll}
\hline Total & 1657 & $100 \%$ & 313 \\
\hline
\end{tabular}

Fuente: elaboración propia.

\section{Instrumento}

Para la recolección de información se construyó por medio de Google Formsun cuestionario en formato electrónico con ocho (8) preguntas cerradas, las cuales se enfocaron en el conocimiento y uso de las herramientas tecnológicas brindadas por la universidad por parte de la población objetivo. Este tipo de instrumento permite que el encuestado responda de manera rápida y específica, facilitando el procesamiento de datos por parte del investigador, que solo debe enfocarse en el análisis de estos.

\section{TIC en la educación}

\section{Importancia}

Con el propósito de lograr un entendimiento de las TIC y su importancia en la educación, primero se debe acudir a su conceptualización. Bajo la visión de Heinze et al. (2017) las TIC son definidas como un conjunto de tecnologías que permiten almacenar, recuperar, procesar y comunicar la información, las cuales tienen tiene una alta incidencia en diversos espacios de la sociedad. Desde el punto de vista tecnológico, las TIC tienen las siguientes características (Meneses, 2007): a) Inmaterialidad, que se refiere a que su materia prima es la información, la cual es representada por tipos de códigos lingüísticos para su transmisión, b) Interactividad, ya que permiten una interacción entre usuario y la máquina, c) Instantaneidad, pues la información está disponible de manera casi instantánea, d) Innovación, porque elevan la calidad y el sonido en el contenido multimedia, e) Digitalización de la imagen y sonido, facilitándola manipulación y distribución de los datos, f) Automatización e interconexión, puesto que pueden funcionar independientemente de las arquitecturas tecnológicas $y, g$ ) Diversidad, en las funciones que pueden desempeñar. Del mismo modo, las TIC cumplen un papel pedagógico que permite: a) promover conocimientos y las habilidades, b) optimizar procedimientos establecidos $y, c)$ crear nuevos modelos por medio de la innovación de los procedimientos (Fabres, Libuy y Tapia, 2014).

Nuestra sociedad se enfrenta a cambios constantes impulsados por el desarrollo de la tecnología y las nuevas formas de comunicación que esta genera. Como resultado de los avances tecnológicos, los individuos disponen de mayores formas de comunicación. Esto ha cambiado la forma de relacionarnos y la forma en que enfrentamos el mundo, incluyendo la forma de aprender. La integración entre aprendizaje y TIC son el sustento para el nacimiento de una sociedad de la información y el conocimiento, en la que instituciones educativas de 
cualquier nivel se ven de cierta forma obligadas a participar ante una influencia de la sociedad que requiere de nuevos procesos educativos de enseñanza y aprendizaje (Zenteno y Mortera, 2011).

La relación entre TIC y los procesos pedagógicos ha influenciado el escenario educativo, convirtiendo la enseñanza tradicional en algo más dinámico, en el cual las TIC están presentes como factor de innovación (Marín, Ramírez y Maldonado, 2015). Por otra parte, se habla de una nueva economía soportada en la información y el conocimiento, que se ha facilitado gracias a la evolución de las TIC y al fácil acceso a ellas, que mejoran los procesos de una organización (Ramos, 2015). Es por ello que la inclusión de las TIC en los procesos de enseñanza y aprendizaje cada vez se hace más necesaria, permitiendo formar profesionales mejor capacitados para las nuevas demandas en gestión de los datos.

Esto ha originado inevitables modificaciones en los roles de estudiantes y profesores, al alterarse la dinámica tradicional de ambos, así como su interacción y medios de comunicación, abriendo nuevos escenarios que requieren de una reflexión hacia el uso e incorporación de las tecnologías, buscando definir la razón de su incorporación y beneficio (Cabero, 2007).

Los cambios tecnológicos presentes en las nuevas sociedades del conocimiento dejan ver la necesidad que tienen todos los actores involucrados de capacitarse en las nuevas técnicas audiovisuales, que son aplicadas en los procesos de enseñanza. Este es un fenómeno propio de la globalización, la cual se manifiesta en las TIC, al posibilitar la comunicación, la interacción y la interconexión entre las personas e instituciones a nivel mundial, y eliminar las barreras de espacio. Igualmente, si las TIC son utilizadas correctamente, posibilitarán el desarrollo de sociedades incluyentes, fortaleciendo la colaboración y distribución de la información, eliminando la brecha digital en las sociedades menos desarrolladas (Bellei, et al., 2013).

En este aspecto, en los últimos años las acciones de los directores universitarios han sido dirigidas a la implantación de infraestructura tecnológica en las aulas universitarias (ordenadores, proyectores, conexión a internet, plataformas de formación on-line...), facilitando a los maestros nuevos recursos para que plante en su labor docente de manera más dinámica y motivadora, y centrada en el auto-aprendizaje de los alumnos (García-Valcárcel, 2007). A lo anterior debe sumarse la formación profesional docente desde la dimensión pedagógica y didáctica, dirigida al desarrollo de cualidades y capacidades requeridas para el nuevo docente del siglo XXI (Valencia, et al., 2016).

\section{Ventajas de las TIC}

Con el uso de nuevas herramientas tecnológicas se promueven oportunidades en las que los estudiantes pueden realizar simulación de problemas reales y prácticos. Además, la interacción que se da entre los diferentes actores educativos, fomenta el aprendizaje activo al poder participar en forma autónoma o colaborativa en la elaboración de actividades y proyectos mucho más retadores y significativos. 
La utilización de las TIC en las instituciones universitarias se ha transformado en un factor determinante para lograr el cambio y la adaptación a las nuevas formas de hacer y pensar en los diferentes ámbitos de la sociedad. En el aspecto administrativo, han de mejorar la organización de las instituciones educativas. En el sector académico, facilitan el acceso de los estudiantes a la información, así como enriquecen significativamente los contextos educativos.

Con las TIC se facilita la búsqueda de información acerca de temas específicos. Así mismo, favorecen la comunicación estudiante-profesor mediante conferencias y chat con terceros, por medio de plataformas virtuales, que generalmente los docentes utilizan para publicar tareas y temas de debate donde los estudiantes dan y comentan las opiniones de otros (Hidalgo, Salazar y Chile, 2018). Es evidente que este tipo de herramientas ahorran tiempo a profesores y estudiantes, evitando el desplazamiento geográfico hasta la institución para realizar procesos propios de la asignatura como consultar resultados de evaluaciones, o los horarios de tutoría de los profesores (Martínez-Rodrigo y González, 2012). Igualmente, las TIC permiten una interacción total con la información, permitiendo configurar la velocidad, secuencia, cantidad y nivel de dificultad de esta. Además, gracias al gran volumen de datos disponibles, convierten a los usuarios en potenciales productores de más conocimiento (Grande, Cañón y Cantón, 2016).

\section{Aplicación de las TIC}

En el sector educativo hoy en día las nuevas formas de aprendizaje son en gran medida producto del desarrollo de las TIC. Una de estas formas es U-learning, que engloba a todas aquellas de actividades de aprendizaje que son accesibles en cualquier momento y lugar. Dentro de esta metodología se encuentra el E-learning,que es aquella educación en línea en la que docentes y estudiantes convergen en un ambiente digital a través de las nuevas tecnologías, que se basa en las funcionalidades que ofrece Internet. De este tipo de aprendizaje se deriva el $B$-learning y el M-learning. El B-learning o también conocido como aprendizaje combinado, consiste en un método de aprendizaje semipresencial, donde se involucra la presencialidad y actividades virtuales que el estudiante realiza desde casa. Mientras que el $M$ learning es un método de enseñanza y aprendizaje que utiliza dispositivos móviles como Smartphone, tabletas, y todo dispositivo de conectividad inalámbrica facilitando la ubicuidad de los actores (Maquilón, 2018).

\section{Las TIC en la Universidad del Valle}

El método o tendencia de aprendizaje utilizado por la Universidad del Valle y en especial por la sede Buga en los programas de formación tecnológica, profesional y posgraduales es el $B$ learning. Está fundamentado principalmente por la utilización de diversas herramientas como:

- El Campus Virtual: es un entorno virtual de aprendizaje (EVA) implantado en Moodle para la gestión de contenido de aprendizaje y que sirve como herramienta de apoyo al proceso de enseñanza y aprendizaje. Permite a los docentes la inscripción de alumnos en los diferentes cursos bajo su responsabilidad, llevar el control de asistencia a clase en 
donde los estudiantes pueden ver su propio registro, la publicación de material digital como: archivos, presentaciones, videos, enlaces a recursos externos, foros, cuestionarios con retroalimentación instantánea y añadir a los cursos objetos virtuales de aprendizaje (OVA) en forma de paquetes SCORM, AICC o IMS Content Packaging. También, la creación de tecnologías sincrónicas como foros y chats permitiendo la comunicación en tiempo real entre el docente y los estudiantes. Adicionalmente, usando Moodle Mobile, el cual se puede descargar del App Store del dispositivo móvil, se puede acceder al campus virtual usando el celular.

- Correo institucional: la institución proporciona a toda la comunidad académica (estudiantes, docentes, administrativos, directivos y dependencias) una cuenta de correo electrónico bajo el dominio @correounivalle.edu.co, soportada por Gmail. Al ser una cuenta institucional, esta ofrece más y mejores herramientas que no están presentes en una cuenta gratuita, por ejemplo: más capacidad de almacenamiento, acceso a los servicios de Google, medidas de seguridad como firewalls, antivirus y filtros anti-spam, Google Docs y trabajo colaborativo, Calendario, Agenda, y herramientas de enseñanza como Jamboard, Meet y Classroom.

- Licencias y software especializados: es un conjunto de cuatro herramientas de software licenciado especializado para uso de profesores y estudiantes, descritas a continuación: (1) Adobe Creative Cloud, una suite educativa que ofrece una amplia plataforma de aplicaciones de escritorio y móviles para la edición de imágenes, publicación digital e impresa, edición profesional de vídeo y películas, creación de gráficos vectoriales y creación de prototipos para sitios web, entre otros. (2) Clarity English, recursos en línea en idioma inglés para instituciones educativas, dentro de los cuales se tiene acceso a: (a) Tense Buster dirigido a la comprensión de la gramática desde el Nivel Elemental hasta el Nivel Avanzado, (b) Active Reading enfocado a la lectura, (c) Practica/Writing enfocado a la escritura y, por último (d) Clear Pronunciation, el cual permite reconocer y pronunciar una gran cantidad de fonemas en inglés. El acceso a esta Suite se hace por medio de la cuenta de correo institucional de los docentes y estudiantes, y desde cualquier dispositivo conectado a Internet, bien sea dentro o fuera de la institución. (3) Wolfram Mathematica, un software utilizado en diversas áreas como la ingeniería, la matemática y ciencias computacionales, y que también es un lenguaje de programación de propósito general. Para hacer uso de Mathematica se debe crear una cuenta en el portal de usuarios de Wolfram con la cuenta de correo electrónico institucional y solicitar la clave de activación, según el tipo de vinculación con la universidad. (4) IBM SPSS Statics Premium, software estadístico para la recolección y análisis de datos utilizado en el sector de negocios y de investigación (Dintev.a, s.f).

- Bases de Datos Académicas: son bases de datos de libros y revistas indexadas en formato electrónico, de carácter científico, en una gran variedad de áreas del conocimiento. El acceso se realiza por medio del código estudiantil o identificación el docente, y una contraseña asignada por el departamento de bibliotecas de la universidad. 
- Caja de herramientas: selección de aplicaciones que ayudan a potenciar las labores de enseñanza y aprendizaje tanto virtuales como presenciales. Pueden articularse con otros servicios tecnológicos, como lo son el Campus Virtual y las Bases de Datos Académicas. Las herramientas están clasificadas de acuerdo al tipo de recurso educativo y actividades didácticas que permiten realizar, dentro de las cuales se pueden mencionar: comunicación, creación multimedia, evaluación del aprendizaje, gamificación, investigación académica, organizadores gráficos y trabajo colaborativo (Dintev.b, s.f).

- Recursos abiertos: conjunto de cursos de formación disponible para toda la comunidad universitaria en áreas como la matemática, física, ciencias naturales y la comprensión y producción de textos escritos (Campus Virtual, s.f).

\section{Resultados y discusión}

A continuación, se presentan de forma consolidada los resultados obtenidos.

Ante la pregunta que buscaba apreciar si el uso de las TIC en la Universidad del Valle sede Buga en los procesos de enseñanza y aprendizaje son parte fundamental de la formación profesional. Los resultados agrupados por facultades son los siguientes:

Tabla 2.

TIC en procesos de enseñanza y aprendizaje y su relación con la formación profesional

\begin{tabular}{|c|c|c|c|c|c|c|c|c|c|c|}
\hline & \multicolumn{2}{|c|}{ Ingeniería } & \multicolumn{2}{|c|}{$\begin{array}{c}\text { Ciencias } \\
\text { Administración }\end{array}$} & \multicolumn{2}{|c|}{ Psicología } & \multicolumn{2}{|c|}{$\begin{array}{c}\text { Artes } \\
\text { Integradas }\end{array}$} & \multicolumn{2}{|c|}{ Total Muestra } \\
\hline & \# & $\%$ & $\#$ & $\%$ & \# & $\%$ & \# & $\%$ & $\#$ & $\%$ \\
\hline Sí & 75 & 97 & 121 & 95 & 26 & 90 & 77 & 96 & 299 & 96 \\
\hline No & 2 & 3 & 6 & 5 & 3 & 10 & 3 & 4 & 14 & 4 \\
\hline
\end{tabular}

Como se puede observar en la Tabla 2, para 299 estudiantes, que representan el $96 \%$ de la muestra, el uso de las TIC es fundamental en su formación profesional. Esto evidencia una marcada tendencia de los estudiantes, sobre todo en carreras enfocadas al sector empresarial, tales como Ingeniería Industrial, Administración de Empresas o Comunicación Social y Periodismo, donde se presenta el porcentaje más alto por facultad con $97 \%$, 95\% y un $96 \%$ respectivamente, a utilizar herramientas tecnológicas que ayuden o complementen su formación y los preparen de la mejor manera para su vida profesional, lo que confirma que las nuevas economías están fuertemente relacionadas con las TIC. 
En cuanto a la pregunta sobre el uso de las herramientas tecnológicas proporcionadas por la Universidad del Valle, se buscaba determinar si se capacita y motiva por parte de los docentes y la sede en general, en el uso de tales herramientas para los procesos de aprendizaje. Los resultados agrupados por facultades son los siguientes:

Tabla 3.

Capacitación y motivación en el uso de TIC en procesos de enseñanza y aprendizaje

\begin{tabular}{|c|c|c|c|c|c|c|c|c|c|c|}
\hline & \multicolumn{2}{|c|}{ Ingeniería } & \multicolumn{2}{|c|}{$\begin{array}{c}\text { Ciencias } \\
\text { Administración }\end{array}$} & \multicolumn{2}{|c|}{ Psicología } & \multicolumn{2}{|c|}{$\begin{array}{c}\text { Artes } \\
\text { Integradas }\end{array}$} & \multicolumn{2}{|c|}{ Total } \\
\hline & \# & $\%$ & \# & $\%$ & \# & $\%$ & \# & $\%$ & \# & $\%$ \\
\hline $\mathrm{Si}$ & 50 & 65 & 82 & 65 & 10 & 34 & 49 & 61 & 191 & 61 \\
\hline No & 27 & 35 & 45 & 35 & 19 & 66 & 31 & 39 & 122 & 39 \\
\hline
\end{tabular}

Los resultados anteriores permiten determinar que, si bien es cierto que para 191 estudiantes, que representan el $61 \%$ de la muestra total, existe capacitación y se motiva el uso de las TIC en los procesos de aprendizaje, no es el mejor de los resultados. Reflejándose un porcentaje muy bajo en los estudiantes de la carrera de Psicología, donde solo el 34\% considera que se capacita y motiva por parte de la institución y los docentes hacia el uso de estas herramientas (Tabla 3).

Este mismo fenómeno se presentó al indagar acerca del conocimiento de los beneficios de utilizar el correo institucional proporcionado por la Universidad del Valle, donde los resultados reflejaron que 210 estudiantes, que representan el $67 \%$ de la muestra total, conocen tales beneficios, siendo un porcentaje relativamente bueno. En este sentido, no se debe pasar por alto que no solo los docentes, sino todos los actores involucrados en la educación requieren de unas capacidades específicas en aspectos técnicos, pedagógicos y metodológicos para hacer frente a estos nuevos desafíos que les ayuden a realizar la adaptación e integración de las TIC en el aula (Mirete, 2010).

Respecto a la frecuencia de uso, se preguntó sobre la cantidad de veces que se utilizan las herramientas tecnológicas brindadas por la Universidad del Valle en los procesos de aprendizaje. Los resultados son los siguientes: 
Figura1.

Frecuencia de uso de las herramientas tecnológicas

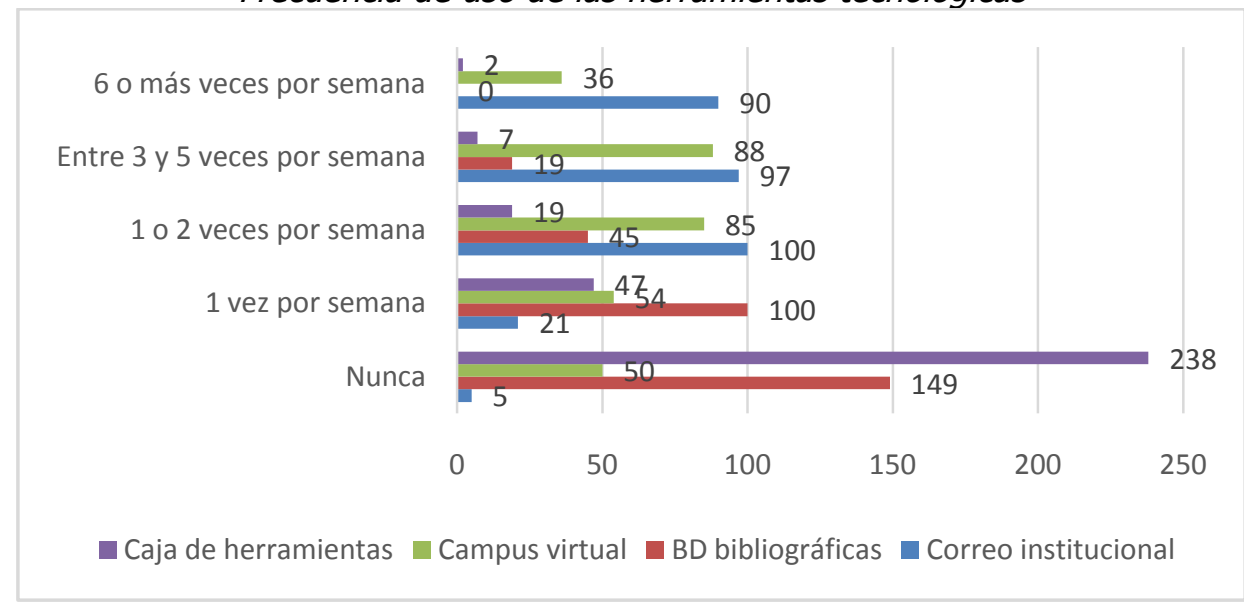

Fuente: elaboración propia

Lo primero a considerar de los resultados anteriores es que se identificó cuáles son las herramientas más utilizadas por parte de los estudiantes, tomando como frecuencia de uso 30 más veces en la semana. El correo institucional, con un $60 \%$, fue el más utilizado. Cabe señalar que, aunque el campus virtual es uno de los pilares fundamentales en los procesos de $B$ learning, solo el $40 \%$ de los estudiantes admitieron que lo utilizan 3 o más veces en la semana (Tabla 4), lo cual es un porcentaje aceptable, que posiblemente es el efecto de la falta de conocimiento y capacitación de los docentes con respecto a los beneficios de la herramienta.

Tabla 4.

Herramientas Tecnológicas más utilizadas en procesos de enseñanza y aprendizaje

\begin{tabular}{lcr}
\hline \multirow{2}{*}{$\begin{array}{c}\text { Frecuencia de uso / } \\
\text { Herramientas }\end{array}$} & \multicolumn{3}{c}{$\begin{array}{c}\text { 3 o más veces en la } \\
\text { semana }\end{array}$} \\
\cline { 2 - 4 } Correo institucional & $\#$ & \% \\
\hline BD bibliográficas & 187 & 60 \\
\hline Campus virtual & 19 & 6 \\
\hline Caja de herramientas & 124 & 40 \\
\hline
\end{tabular}

Fuente: elaboración propia

Así mismo se estableció que los recursos de caja de herramientas y bases de datos bibliográficas presentaron los porcentajes más altos en cuanto a lo no utilización de estos, con un porcentaje del 76\% que representa a 238 estudiantes, y un $48 \%$ que representa a 149 estudiantes respectivamente, siendo la facultad de Artes Integradas, seguida por la facultad de Ciencias Administración donde se presentaron los porcentajes más altos (Tabla 5). 
Tabla 5.

Frecuencia de Uso - Caja de Herramientas

\begin{tabular}{lcc}
\multicolumn{1}{c}{$\begin{array}{c}\text { Calificación / } \\
\text { Facultad }\end{array}$} & \multicolumn{2}{c}{ Nunca } \\
\cline { 2 - 3 } & $\#$ & $\%$ \\
\hline Ingeniería & 47 & 61 \\
\hline Ciencias Administración & 97 & 76 \\
\hline Psicología & 22 & 76 \\
\hline Artes Integradas & 72 & 90 \\
\hline & Total & 238 \\
\hline
\end{tabular}

Fuente: elaboración propia

Tabla 6.

Frecuencia de Uso - Bases de Datos Bibliográficas

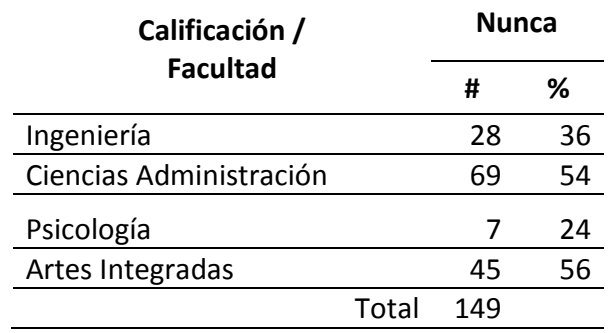

Fuente: elaboración propia

También, con respecto a la frecuencia de uso, se preguntó sobre la cantidad de veces que se utilizan las aplicaciones licenciadas brindadas por la Universidad del Valle, arrojando los siguientes resultados sobre la muestra total:

Figura 2.

Frecuencia de uso de las aplicaciones licenciadas

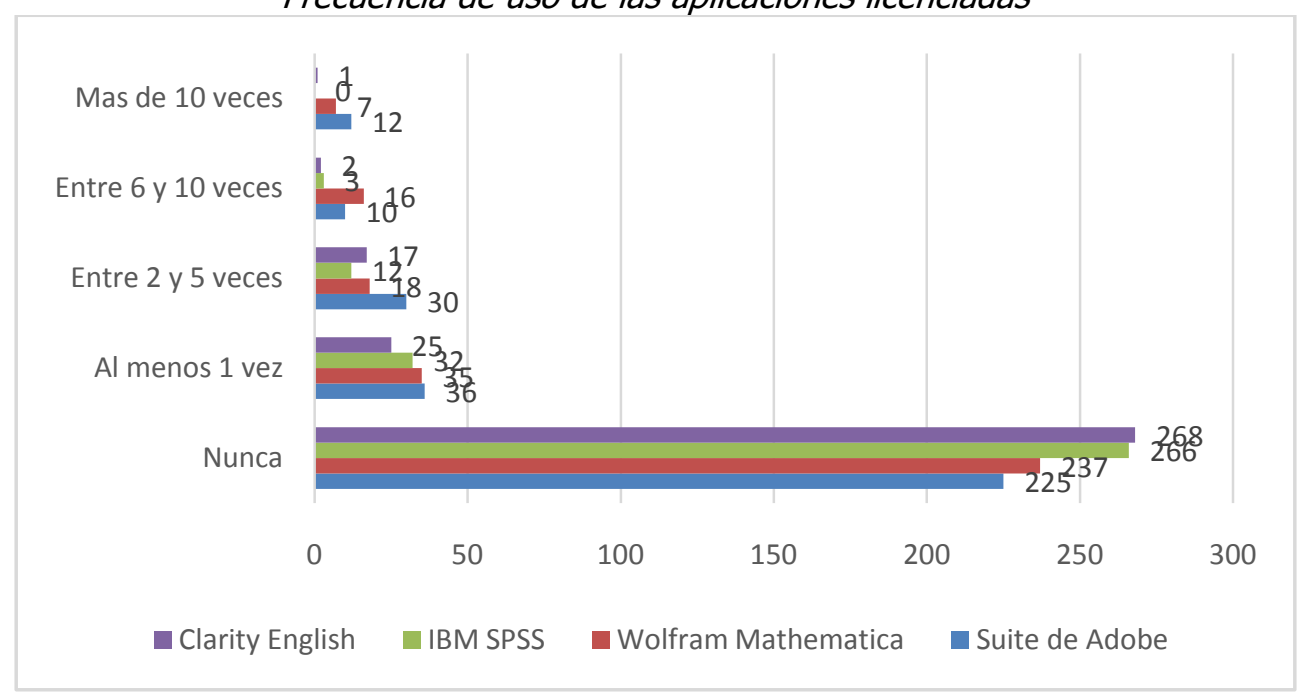

Fuente: elaboración propia 
De lo anterior se puede concluir que, aunque la universidad se encuentra a la vanguardia tecnológica y realiza enormes esfuerzos presupuestales en la compra de software, este no es aprovechado por los docentes y estudiantes, estos resultados se comprobaron en las cuatro aplicaciones evaluadas, en las cuales el porcentaje de no uso oscila entre el $72 \%$ y el $86 \%$ (ver Figura 2), posiblemente debido a factores como: desconocimiento de que la institución cuenta con esta tecnología, falta de capacitación o adaptación de los contenidos curriculares para que se utilicen estas tecnologías. En tal sentido, es claro que las TIC en el ámbito educativo posibilitan mejoras en el funcionamiento interno de las instituciones, y en la docencia permite la entrada en nuevos segmentos de mercado que antes no se podían alcanzar, pero que en ningún momento se trata de una ventaja por sí misma, si tales tecnologías son desaprovechadas por cuestiones de conocimiento y capacitación (Castel, 2018).

En este punto es importante mencionar que la integración de las TIC en las prácticas pedagógicasno solo trata de implantar tecnología en los establecimientos educativos, también es cualificar a los docentes en el uso de las tecnologías y es solo entonces cuando los estudiantes aprenden con la tecnología (Pulido y Najar, 2014).

En relación con los anteriores resultados, a los estudiantes se les preguntó si considerabanque se debería de fomentar más el uso de las aplicaciones licenciadas en los diferentes cursos realizados en la Universidad del Valle sede Buga, a lo cual el 99\% respondió que Sí, lo cual es un reflejo de la disposición por parte de los estudiantes a utilizar TIC que mejoren sus procesos educativos y los preparen para resolver problemas en la vida profesional haciendo uso de estas herramientas. Este resultado confirma estudios anteriores que consideraban que los estudiantes tienen interés en el uso de tecnologías de información para la construcción de su propio conocimiento, demandando estrategias y recursos que promuevan la creatividad y la imaginación (Martínez, Burbano y Burbano, 2019).

Finalmente, se pidió a los estudiantes que calificaran de 1 a 5 , donde 1 es la mínima calificación y 5 es la máxima, la importancia que tienen las TIC en los procesos de enseñanza y aprendizaje. En la Figura 3 se omite la calificación 1 y 2 al no estar presentes en las respuestas. Los siguientes son los resultados agrupados por facultades: 
Figura 3.

Importancia del uso de las TIC en la educación

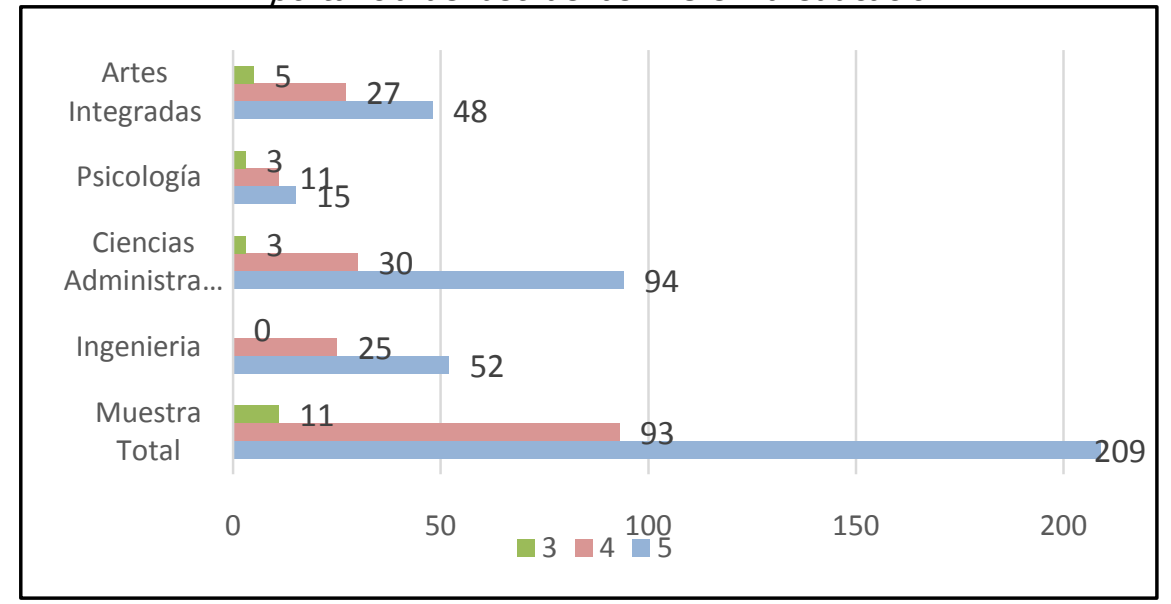

Fuente: elaboración propia.

Nuevamente se ve reflejado el interés y el grado de percepción de los estudiantes acerca de la importancia que tienen las TIC en su proceso educativo, ya que alrededor del $96 \%$ dieron la calificación más alta. En tal sentido, los nuevos modelos de la economía y de diversas áreas, se sustentan en la formación de competencias que le permitan al estudiante, por medio de su aprendizaje, prepararse para la incorporación a situaciones más dinámicas, que demandan una gestión del conocimiento, y aplicación de aprendizajes y habilidades (Castillo y Comelin, 2016).

\section{Conclusiones}

Hoy en día, la utilización de nuevas herramientas tecnológicas permite a la sociedad mejorar la calidad de vida de los ciudadanos y a diversos sectores de la economía optimizar y perfeccionar de manera sustancial sus procesos.

La educación no es ajena a la obligación de implantar nuevas TIC, que permitan que los modelos tradicionales educativos se alineen con las actuales tendencias de conocimiento, renovando así los métodos de enseñanza y la forma de aprendizaje de los estudiantes, para que se adapten a los nuevos requerimientos de los mercados laborales. Pero esta adopción implica reformas económicas e institucionales que garanticen la correcta implementación no solo de tecnologías, también de las habilidades gerenciales, organizativas y pedagógicas necesarias para su uso, que satisfagan las exigencias de un entorno que cada vez es más cambiante.

La presente investigación evidenció que si bien es cierto la Universidad del Valle en la sede de Buga ofrece una gran cantidad de herramientas tecnológicas dirigidas a todas las áreas de estudio, en su gran mayoría aplicaciones de software que están a la vanguardia, existe una marcada falta de conocimiento de las mismas, tanto por estudiantes como por los docentes, y 
la manera en que estas se pueden adoptar en los procesos de enseñanza y aprendizaje. Así mismo, se comprobó que los estudiantes de la sede entienden la importancia y ven la necesidad de la incorporación de las TIC en su proceso formativo, pero revelan que su uso en general no cumple totalmente con las expectativas.

En general, los resultados obtenidos demuestran que las herramientas brindadas por la Universidad del Valle se están quedando inutilizadas y algunas resultan prácticamente desconocidas. Por lo anterior, se debe alentar a la sede y en especial a directores de programas académicos y dependencias de apoyo a la docencia, a establecer estrategias en cuanto al uso de estas herramientas y brindar cursos o seminarios de capacitación no solo dirigidos a los estudiantes, también a los docentes, que les ayuden a involucrarlas en los contenidos curriculares, ya que puede presentarse la situación en la cual el docente no motiva la utilización de las TIC porque simplemente no las conoce, o las conoce pero no las sabe utilizar, o porque la institución no cuenta con las herramientas adecuadas que se adapten a sus procesos pedagógicos.

\section{Referencias}

Abarca, Y. (2015). El uso de las TIC en la educación universitaria: motivación que incide en su uso y frecuencia. Revista de Lenguas Modernas. (22), 335-349. https://revistas.ucr.ac.cr/index.php/rlm/article/download/19692/19771

Bellei, C., et al. (2013). Situación Educativa de América Latina y el Caribe: Hacia la educación de calidad para todos al 2015.Centro de Investigación Avanzada en Educación de la Universidad de Chile. http://www.unesco.org/new/fileadmin/MULTIMEDIA/FIELD/Santiago/images/SITIEDespanol.pdf

Bustos, O., y Parra, K. (2019). Integración de las TIC en la enseñanza de la historia en educación media superior. Revista Boletín Redipe, 8(1), 106-113. https://dialnet.unirioja.es/descarga/articulo/6804247.pdf

Cabero, J. (2007). Las necesidades de las TIC en el ámbito educativo: oportunidades, riesgos y necesidades. http://investigacion.ilce.edu.mx/tyce/45/articulo1.pdf

Campus Virtual. (s.f.). Recursos abiertos. Dirección de Nuevas Tecnologías y Educación Virtual. https://campusvirtual.univalle.edu.co/

Castel, A. (2018). La integración de las TIC en los procesos educativos y organizativos. Educar em Revista, 34(69), 325-339. https://dx.doi.org/10.1590/0104-4060.57305 
Castillo, L., y Comelin, A. (2016). Integración de TIC en la formación de competencias profesionales para entrevistas con niños en trabajo social. Revista Contextos. (36), 13-31. https://dialnet.unirioja.es/descarga/articulo/6086945.pdf

Castro, S., Guzmán, B., y Casado, D. (2007). Las Tic en los procesos de enseñanza y aprendizaje. $\quad$ Laurus, 13(23), 213-234. https://www.redalyc.org/articulo.oa?id=761/76102311

Dintev.a. (s.f.). Licencias y software especializado. Dirección de Nuevas Tecnologías y Educación Virtual. https://sites.google.com/correounivalle.edu.co/licenciasysoftwareunivalle/p\%C3\%A1ginaprincipal?authuser $=0$

Dintev.b. (s.f.). Herramientas para la Innovación Educativa. Dirección de Nuevas Tecnologías y Educación Virtual. http://svtie.univalle.edu.co/

Fabres, J., Libuy, D., y Tapia, P. (2014). Análisis del uso de las tecnologías de la información y la comunicación en los establecimientos educacionales de Chile: caso del colegio Santo Tomás de la Comuna de Nuñoa. http://repositorio.uchile.cl/bitstream/handle/2250/116586/Fabres\%2C\%20Juan\%20Carlos .pdf?sequence $=1$ \&isAllowed $=y$

Garcés, E., Garcés, E., y Alcívar, O. (2016). Las tecnologías de la información en el cambio de la educación superior en el siglo XXI: Reflexiones para la práctica. Revista Universidad y Sociedad, 8(4), 171-177. http://scielo.sld.cu/scielo.php?script=sci_arttext\&pid=S221836202016000400023\&lng=en\&tlng=en

García-Valcárcel, A. (2007). Herramientas tecnológicas para mejorar la docencia universitaria. Una reflexión desde la experiencia y la investigación. Rev. Iberoamericana de Educación a Distancia. 10(2). https://doi.org/10.5944/ried.2.10.996

Grande, M., Cañon, R., y Cantón, I. (2016). Tecnologías de la información y la comunicación: evolución del concepto y características. International Journal of Educational Research $\begin{array}{llll}\text { and Innovation (IJERI), 218-230. } & \text { (6), }\end{array}$ https://www.upo.es/revistas/index.php/IJERI/article/view/1703/1559

Heinze, G., Olmedo, V., y Andoney, J. (2017). Uso de las tecnologías de la información y comunicación (TIC) en las residencias médicas en México. Acta médica Grupo Ángeles, 15(2), 150-153. http://www.scielo.org.mx/scielo.php?script=sci_arttext\&pid=S1870$72032017000200150 \& \operatorname{lng}=e s \&$ tlng=es

Hernández, M. (2017). Impacto de las TIC en la educación: Retos y Perspectivas. Propósitos y Representaciones, 5(1), 325 - 347. http://dx.doi.org/10.20511/pyr2017.v5n1.149 
Hernández, R., Fernández, C., y Baptista, P. (2014). Metodología de la Investigación. 6a ed. Mc Graw Hill.

Hidalgo, F., Salazar, M., y Chile, S. (2018). El Uso de las Tecnologías Educativas y su Impacto en la Formación de los Profesionales de la Educación. Opuntia Brava, 10(1), 296-302. https://doi.org/10.35195/ob.v10i1.76

Islas, C. (2017). La implicación de las TIC en la educación: Alcances, Limitaciones y Prospectiva. RIDE. Revista Iberoamericana para la Investigación y el Desarrollo Educativo. 8(15), 1-16. http://dx.doi.org/10.23913/ride.v8i15.324

Lasso, L. y Sánchez-Medina, I. (2019). Implantación de una plataforma de aprendizaje para el curso de matemáticas grado noveno en la Institución San Vicente, Colombia. Espacios. 40(21), 28. http://www.revistaespacios.com/a19v40n21/19402128.html

Marín, V., Ramírez, M. y Maldonado. G. (2015). Valoraciones del profesorado universitario sobre la integración de las TIC en el aula. EDMETIC, 5(1). https://doi.org/10.21071/edmetic.v5i1.4022

Martínez-Rodrigo, E., y González, F. (2012). Renovación, innovación y TIC en el EEES. Rev. ICONO 14(7), 50-63. https://doi.org/10.7195/ri14.v7i3.302

Martínez, J., Burbano, M., y Burbano, E. (2019). Obstáculos y perspectivas al emplear tecnologías de información para enseñar contabilidad. Educación y Humanismo, 21(37), 104-119. https://doi.org/10.17081/eduhum.21.37.3461

Maquilón, E. (2018). Diagnóstico y Percepción de Aplicación de Las Tics Como Herramientas Para La Educación Superior. Espirales: Rev. Multidisciplinaria de Investigación. (18), 1427. https://www.revistaespirales.com/index.php/es/article/view/312/232

Meneses, G. (2007). NTIC, Interacción y Aprendizaje en la Universidad. https://www.tesisenred. net/bitstream/handle/10803/8929/2Lasnuevastecnologiasdelainfor macion.pdf?sequence $=8$

Mirete, A. (2010). Formación docente en Tics. ¿están los docentes preparados para la (r)evolución TIC?.International Journal of Developmental and Educational Psychology, 4(1), 1-16. https://www. redalyc.org/articulo.oa?id=3498/349832327003

Pimienta, R. (2000). Encuestas probabilísticas vs. no probabilísticas. Política y Cultura, (13), 2537. https://www.redalyc.org/articulo.oa?id=267/26701313

Pulido, D., y Najar, O. (2014). Gestión del conocimiento en educación con tic en la transformación de la escuela. Revista Vínculos. 12(1), 41-55. https://doi.org/10.14483/2322939X.10520 
Quiroga, D., Torrent, J., y Murcia, C. (2017). Usos de las TIC en América Latina: una caracterización. Ingeniare. Revista chilena de ingeniería, 25(2), 289-305. https://dx.doi.org/10.4067/S0718-33052017000200289

Ramos, A. (2015). Gestión del conocimiento en el proceso de docencia para instituciones de educación superior. SIGNOS - Investigación en sistemas de gestión. 7(2), 31-43. https://doi.org/10.15332/s2145-1389.2015.0002.02

Severin, E. (2010). Tecnologías de la Información y la Comunicación (TIC's) en Educación. http://repositorio.minedu.gob.pe/handle/123456789/3394

Valencia, T., et al. (2016). Competencias y estándares TIC desde la dimensión pedagógica: Una perspectiva desde los niveles de apropiación de las TIC en la práctica educativa docente. http://www.unesco.org/new/fileadmin/MULTIMEDIA/FIELD/Santiago/pdf/Competenciasestandares-TIC.pdf

Zenteno, A. y Mortera, F. (2011). Integración y apropiación de las TIC en los profesores y los alumnos de educación media superior. Apertura. Revista de innovación educativa. 3(1), 1-21. https://www.udgvirtual.udg.mx/apertura/index.php/apertura/article/view/193/208 